\title{
A TWO-DIMENSIONAL MODELLING FOR HUMAN HIP STRESS ANALYSIS
}

\author{
XiShi Wang ${ }^{1}$, TianYing Wang ${ }^{1}$, FuChuan JiAng ${ }^{1}$, YiXIang Duan ${ }^{2}$ \\ 'Dept of Mathematics and Physics, QingDao Institute of Architecture and Engineering, \\ ${ }^{2}$ Dept of Orthopaedics, QingDao Hospital, \\ QingDao, P.R. China
}

\begin{abstract}
This paper, based on the elasticity theory, a two dimensional modelling for the hip stress analysis in the sagittal plane is presented. Though the stress, in the normal human hip, is distributed over the entire crossection of the femur head, the main activities of the human being are limited in the sagittal plane. Moreover, a 2-D modelling for the stress analysis is much simpler and easier than 3-D one; especially it can be combined with the clinical standard anterior posterior rentgenographs. These predicate that it is useful and necessary to develop a 2-D modelling for the hip stress analysis. The validation of current modelling is verified by examining an example. In other words, the present modelling can be readily applied in clinical practice to estimate the peak stress in the most frequent body positions of everyday activities.
\end{abstract}

Biomed Eng Appl Basis Comm, 2004 (February); 16: 32-36.

Keywords: Hip stress analysis, 2-D modelling, peak stress, stress distribution

\section{INTRODUCTION}

Abnormal mechanical stress upon hip joint cartilage is one of the main causes of osteoarthritis. It is well recognized that intrinsic pathomechanical changes in articulator cartilage depends upon local stress levels rather than upon global joint loading. Therefore, it is essential to estimate the stress distribution and its peak value on the hip joint surface in order to understand the pathomenchanical changes in the hip joint cartilage. In another aspect, the treatments of osteoarthritis of hip joint vary from conservative rehabilitative exercises to surgical treatments such as osteotomies or total hip

Received: Dec 5, 2003; Accepted: Dec 25, 2003

Correspondence: XiShi Wang, Professor

Dept of Mathematics and Physics, QingDao Institute of Architecture and Engineering, No.11, FuShun Rd., QingDao, 266033, P.R. China

E-mail: wang_xishi@hotmail.com arthroplasties. Therefore, the estimation of hip joint stress distribution and its peak calue is very useful for both preoperative planning and postoprerative rehabilitation. Furthermore, knowing the hip stress distribution is also very helpful in understanding the mechanics of the normal hip and understanding the pathology of the articular cartilage of the hip.

In 1970's, Hamacher et al [1-3] developed a modelling for calculating the resultant hip force; Legal et al [4-7], based on previous work [8-11], developed a method for calculation of the hip joint stress distribution for a specific case. Later, various modellings for estimation of the hip joint stress distribution were presented [12-16]. The recent modellings to study hip joint stress distribution are mainly based on an important assumption, which adopted a cosine function form as the stress distribution at the hip joint [17-21].

Although the stress, in the normal human hip, is distributed over the entire crossection of the femur head, the main activities of the human being are limited in the sagittal plane. Moreover, a 2-D 
modelling for the stress analysis is much simpler and easier than 3-D one; especially it can be combined with the clinical standard anterior posterior rentgenographs. All of these predicate that it is useful and necessary to establish 2-D modelling for the hip stress analysis.

In this work, a two dimensional modelling for the human hip stress analysis in the sagittal plane is presented. In order to verify the validation of current two dimensional modelling, an example is examined. The results show that the prediction from the present modelling is good agreement with those reported in literatures. This shows the validity of the current 2-D modelling.

\section{METHODS}

\subsection{Modelling Description}

From the anatomy, it can be known that the human femur head is hemisphere, its surface is the smooth, coated with cartilage in the fresh state. Therefore, in this study, the femur head is modeled as a hemispherical shape, in which the cartilaginous layer is modeled as an elastic layer, while the femur head under the cartilaginous layer is modeled as a rigid body. Thus, a two dimensional modelling on the human hip in the sagittal plane and its coordinate system can be constructed as shown in Figure 1. In order to let the present modelling have practicality, the several assumptions are made as following: 1) The cartilaginous layer consists of isotropic linearity elastic material; 2) The characteristic lengths of cartilaginous layer are much greater than their thickness in the dimensions; 3 ) The femur head underlying the cartilaginous layer is modeled the rigid body. Based on the previous assumptions, the human hip joint can be predigested as the elastic cartilaginous layer covering on the femur head with the rigid body behavior, as shown in Figure 1.

\subsection{Coordinate System and Analytical Solution}

The coordinate system for the current two dimensional modelling at the hip joint is constructed as shown in Fig. 1. The point $O$ is the origin of the coordinate system. The inner radii and the thickness of cartilaginous layer are, respectively, $\boldsymbol{R}$ and $\boldsymbol{a}$. The $\mathrm{P}$ is the hip resultant force, and it is supposed that it acts on the symmetric plane as shown in Figurel. The hip resultant force $\boldsymbol{P}$ can be determined in different phases of a stance period of gait of a person with an implanted total hip endoproathesis [22], or it can be also determined using a 3D mathematical model of an adult human hip in a one-legged stance [16, 23]. The $M$ is an arbitrary point at the cartilaginous layer. The $\alpha$ is the half of conical angle of hemisphere. Thus, in order to

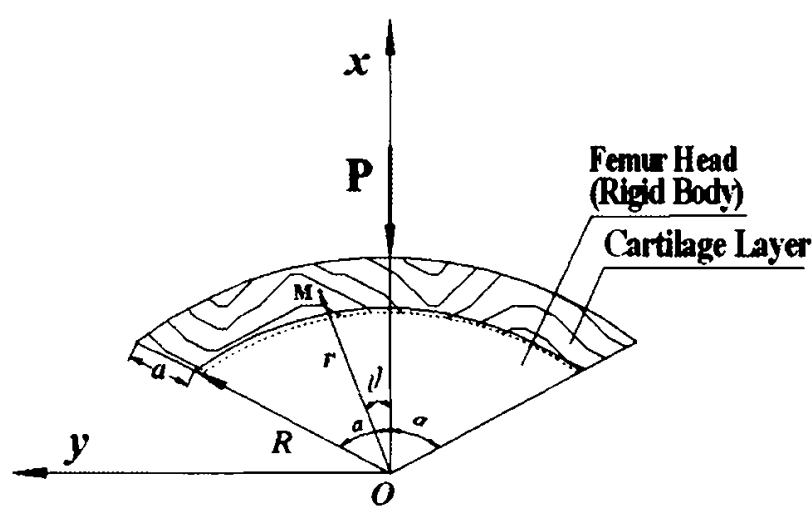

Fig. 1. A two dimensional modelling for the human hip stress analysis in the sagittal plane and its coordinate system.

obtain the problem solution, the polar coordinate system can be employed due to the symmetry. In other words, the current two dimensional modelling is already simplified as a form, in which the plane stress problem is solved under the polar coordinate system. According to the elastic theory, if the stress function is taken as $\varphi$, then the $\varphi$ must satisfies the following equation:

$$
\nabla^{2} \nabla^{2} \varphi=0
$$

where the $\nabla^{2}$ is the Laplace operator.

Since the stress component $\sigma_{r}$ is the function of polar angle $\theta$, and it is in proportion to the distance from the origin point $\boldsymbol{O}$. Therefore, according to the elastic theory, the stress function $\varphi$ can be taken as following form:

$$
\varphi=r^{3} f(\theta)
$$

Substituting equation (2) into (1), then the following equation must be held:

$$
\frac{d^{4} f}{d \theta^{4}}+10 \frac{d^{2} f}{d \theta^{2}}+9 f=0
$$

From equation (3) we have:

$$
f(\theta)=A \cos \theta+B \sin \theta+C \cos 3 \theta+D \sin 3 \theta
$$

where the A, B, C and D are, respectively, the constants to be determined by the boundary conditions.

Thus, the stress components can be obtained as following: 


$$
\left\{\begin{aligned}
\sigma_{r} & =\frac{1}{r} \frac{\partial \varphi}{\partial r}+\frac{1}{r^{2}} \frac{\partial^{2} \varphi}{\partial^{2} \theta} \\
& =2 r(A \cos \theta+B \sin \theta-3 C \cos 3 \theta-3 D \sin 3 \theta) \\
\sigma_{\theta} & =\frac{\partial^{2} \varphi}{\partial r^{2}} \\
& =6 r(A \cos \theta+B \sin \theta+C \cos 3 \theta+D \sin 3 \theta) \\
\tau_{r \theta} & =\tau_{\theta r}=-\frac{\partial}{\partial r}\left(\frac{1}{r} \frac{\partial \varphi}{\partial \theta}\right) \\
& =2 r(A \sin \theta-B \cos \theta+3 C \sin 3 \theta-3 D \cos 3 \theta)
\end{aligned}\right.
$$

\subsection{Boundary Conditions}

There are four boundaries in the modelling. At every boundary, they must satisfy the force boundary conditions. They are:

$$
\begin{aligned}
& \text { 1) at } r=R:\left.\quad \int_{-\alpha}^{\alpha} \sigma_{r}\right|_{r=R} R \cos \theta=-P \text {; } \\
& \left.\int_{-\alpha}^{\alpha} \sigma_{r}\right|_{r=R} R \sin \theta=0 \\
& \left.\tau_{r \theta}\right|_{r=R}=\left.\tau_{\theta r}\right|_{r=R}=0 \text {; } \\
& \text { 2) at } r=R+a:\left.\quad \tau_{r \theta}\right|_{r=R+a}=\left.\tau_{\theta t}\right|_{r=R+a}=0 \\
& \text { 3) at } \theta= \pm \alpha:\left.\quad \int_{R}^{R+a} \sigma_{\theta}\right|_{\theta= \pm \alpha} d r=0 \text {; } \\
& \left.\tau_{r \theta}\right|_{\theta= \pm \alpha}=\left.\tau_{\theta r}\right|_{\theta= \pm \alpha}=0 \text {; }
\end{aligned}
$$

From boundary conditions (B-1) and (B-4), the constants $A, B, C$ and $D$ can be determined as following:

$$
\left\{\begin{array}{l}
A=-\frac{P \cos 3 \alpha}{N} \\
B=0 \\
C=\frac{P \cos \alpha}{N} \\
D=0
\end{array}\right.
$$

where

$$
N=\frac{1}{R^{2}\left(6 \sin 2 \alpha \cos ^{3} \alpha+2 \alpha \cos 3 \alpha+\sin 2 \alpha \cos 3 \alpha\right)}(7)
$$

Thus, substituting equations (6) and (7) into equation (5), the stress components for the current modelling can be presented as following:

$$
\left\{\begin{array}{l}
\sigma_{r}=-2 P \frac{\cos 3 \alpha \cos \theta}{N} r \\
\sigma_{\theta}=6 P \frac{\cos \alpha \cos 3 \theta-\cos 3 \alpha \cos \theta}{N} r \\
\tau_{r \theta}=\tau_{\theta}=0
\end{array}\right.
$$

From the equations ( 8 ), it can be seen that the stress components are the symmetric function of the polar angle ; while the boundary conditions which the shear stress equals zero is automatically satisfied. This is the result of what it is predicated from the present modelling(see Figure 1). Thus, the equations (8) can be used to study the hip joint stress distribution and peak stress.

\section{RESULTS AND DISCUSSIONS}

In the previous section, a two dimensional modelling for the human hip joint in the sagittal plane was successfully established. In order to verify the validation of current two dimensional modelling, here an example is examined. The relative parameters are taken as[24]: human body weight: $70 \mathrm{~kg} ; \mathrm{R}=21 \mathrm{~mm}$; $\mathrm{a}=1 \mathrm{~mm} ; \alpha=30^{\circ}$. Then the calculated result for the peak stress is $-7.1176 \mathrm{MPa}$. This result is good agreement with those reported in the literatures for peak stress in the cartilaginous layer of femur head, such as: $6.8 \mathrm{MPa}[25], 8.8 \mathrm{MPa}[26], 4.9-9.6[27]$, 6-10MPa[28], 7.5-9.0MPa[29], 7.7 MPa[30], etc. This shows directly the validity of the current two dimensional modelling for the hip joint stress analysis.

\section{CONCLUSIONS}

The peak stress, the weight bearing area and the stress distributions of the articular contact at the human hip joint are thought to be most important factors, which determine the location of the degenerative foci that later result in degenerative damage and in the development of osteoarthritis. So far, however, these key factors are mainly determined by measured in vitro or vivo. Therefore, it is necessary to develop a predictive modelling for clinical practice. Although the stress, in the normal human hip, is distributed over the entire crossection of the femur head, the main activities of the human being are limited in the sagittal plane. Moreover, a 2-D modelling for the stress analysis is much simpler and easier than 3-D one; especially it can be combined with the clinical standard anterior 
posterior rentgenographs. All of these predicate that it is useful and necessary to establish 2-D modelling for the hip stress analysis. In this work, a two dimensional modelling for the human hip stress analysis in the sagittal plane is presented. In order to verify the validation of current two dimensional modelling, an example is examined. The results show that the prediction from the modelling is good agreement with those reported in literatures. This shows the validity of the current $2-\mathrm{D}$ modelling.

\section{ACKNOWLEDGEMENT}

This work is supported by Natural Science Foundation of ShanDong Province in China(Grand No. Y2003C01).

\section{REFERENCES}

1. Hamacher P, Rosler H: Die Berechnung von Grosse und Richtung der $\mathrm{H}$ ftgelenksresultierenden im Einzelfall. Arch Orthop Trauma Surg 1971(70): 26-35.

2. Hamacher $P, R$ sler $H$ : Ergebnisse der Berechnung von Grosse und Richtung der $\mathrm{H}$ ftgelenksresultierenden im Einzelfall. Arch Orthop Trauma Surg, 1972(72): 94-106.

3. Hamacher P, R sler H: Belastungsdiagramme bei H ftgelenks-erkrankungen. Z Orthop. 1974(112): 176-186.

4. Legal $\mathrm{H}$ : Introduction to the biomechanics of the hip. In: $T$ nnis D (ed) Congenital dysplasia and dislocation of the hip. Springer Verlag, Berlin Heidelberg New York, 1987, pp 26-57.

5. Legal H, Ruder H: (1977) Zur biostatischen Analyse des H ftgelenks. Z Orthop, 1977(115): 215-234.

6. Legal H, Reinecke M, Ruder H (1978) Zur biostatischen Analyse des $\mathrm{H}$ ftgelenks II. Z Orthop, 1978(116): 889-896.

7. Legal H, Reinecke M, Ruder H (1980) Zur biostatischen Analyse des $\mathrm{H}$ ftgelenks III. Z Orthop, 1980(118): 804-815.

8. Amtmann E, Kummer B: (1968) Die Beanspruchung des menschlichen $\mathrm{H}$ ftgelenks. $\mathrm{Z}$ Anat Entwicklungsgesch. 1968(127): 286-314.

9. Pauwels F: Biomechanics of the normal and diseased hip. Springer Verlag, Berlin Heidelberg New York, 1976.

10. Rösler H, Hamacher P (1972) Die biostatische Analyse der Belastung des Hüftgelenks. Part I. Z Orthop. 1972(110): 67-75.
11. Rösler H, Hamacher P (1972) Die biostatische Analyse der Belastung des $\mathrm{H}$ ftgelenks. Part II. $\mathrm{Z}$ Orthop. 1972(110): 186-196.

12. Antolič V, Kralj-Iglič V, Iglič A, Srakar F: LeggCalve-Perthes disease - stress distribution on the hip joint articular surface after varisation osteotomy. In: Held KD, Brebbia CA, Ciskowski $\mathrm{RD}$, Power $\mathrm{H}$ (eds) Computational biomedicine. Computational Mechanics Publications, Southampton, 1993, pp 255-262.

13. Brinckmann P, Frobin W, Hierholzer E: Stress on the articular surface of the hip joint in healthy adults and persons with idiopathic osteoarthrosis of the hip joint. J. Biomech. 1981(14): 149-156.

14. Genda E, Konishi N, Hasegawa Y, Miura T: A computer simulation study of normal and abnormal hip joint contact pressure. Arch Orthop Trauma Surg. 1995 (114): 202-206.

15. Hipp JA, Sugano N, Millis MB, Murphy SB: Planning acetabular redirection osteotomies based on joint contact pressures. Clin Orthop. 1999(364): 134-143.

16. Iglič A, Kralj-Iglič V, Antolic V, Srakar F, Stanič U: Effect of the periacetabular osteotomy on the stress on the human hip joint articular surface. IEEE Trans Rehab Eng. 1993(1): 207-212.

17. Iglic A, Kralj-Iglic V, Antolic V. Reducing the stress in the articular surface of the hip joint after shifting the upper part of the body towards the painful hip. Acta Chir Orthop Traumatol Czech. 1994(61): 268-70.

18. Ipavec M, Iglic A, Iglic VK, Srakar F. Stress distribution on the hip joint articular surface during gait. Pflugers Arch. 1996, 431 (6): R275-6.

19. Ipavec $M$, Brand RA, Pedersen DR, Mavcic B, Kralj-Iglic V, Iglic A. Mathematical modelling of stress in the hip during gait. J Biomech. 1999, 32 (11): 1229-35.

20. Mavcic B, Antolic V, Brand R, Iglic A, Kralj-Iglic $\mathrm{V}$, Pedersen DR. Peak contact stress in human hip during gait. Pflugers Arch, 2000, 440 (5): R177-8.

21. Daniel M, Antolic V, Iglic A, Kralj-Iglic V. Determination of contact hip stress from nomograms based on mathematical model. Med Eng Phys. 2001, 23 (5): 347-57.

22. Brand, R.A. Hip osteotomies a biomechanical consideration. Journal of the American Academy of Orthopaedic Surgeons 5, 1994, 282-291.

23. Daniel M, Antolic V, Iglic A, Kralj-Iglic V. Determination of contact hip stress from nomograms based on mathematical model. Med Eng Phys 2001; 23 (5): 347-57.

24. Gu Zhihua, Gao Ruiting, The basis of the bone injury biomechanics, Tianjing University Press, 1990, page 103-113. 
25. Rushfeld PD, Mann RW, Harris WH. Influence of cartilage geometry on the pressure distribution in the human hip joint. Science 1979; 204 (4391): 413-5.

26. Brown, T.D., Shaw, D.T. In vitro contact stress distributions in the natural human hip. Journal of Biomechanics. 1983(16); 373-384.

27. Adams D, Swanson SA. Direct measurement of local pressures in the cadaveric human hip joint during simulated level walking. Ann Rheum Dis $1985 ; 44$ (10): 658-66.
28. Maxian TA, Brown TD, Weinstein SL. Chronic stress tolerance levels for human articular cartilage: two nonuniform contact models applied to longterm follow-up of CDH. J Biomech 1995; 28 (2): 159-66.

29. Hak DJ, Hamel AJ, Bay BK, Sharkey NA, Olson SA. Consequences of transverse acetabular fracture malreduction on load transmission across the hip joint. J Orthop Trauma 1998; 12 (2): 90-100.

30. von Eisenhart R, Adam C, Steinlechner M, MullerGerbl M, Eckstein F. Quantitative determination of joint in-congruity and pressure distribution during simulated gait and cartilage thickness in the human hip joint. J Orthop Res 1999; 17 (4): 532-9. 